\title{
Sign-changing solutions and multiplicity results for elliptic problems via lower and upper solutions
}

\author{
Colette De Coster
}

\begin{abstract}
In the first part of this work, we recall variational methods related to invariant sets in $C_{0}^{1}$. In the second part of the work, we consider an elliptic Dirichlet problem in a situation where the origin is a solution around which the nonlinearity has a slope between two consecutive eigenvalues of order larger than 2 and near + infinity the slope of the nonlinearity is smaller than the first eigenvalue. Then we discuss the conditions needed near - infinity in order to ensure the existence of a positive solution and two sign-changing solutions.
\end{abstract}

Mathematics Subject Classification (2000). 35B05, 35J20, 35J60.

\section{Introduction}

In 1982, H. Höfer [17] considered the problem

$$
\begin{aligned}
-\Delta u & =f(u), \quad \text { in } \Omega, \\
u & =0, \quad \text { on } \partial \Omega,
\end{aligned}
$$

where $\Omega \subset \mathbb{R}^{N}$ is a bounded smooth domain, and proved that if $f \in \mathcal{C}^{1}(\mathbb{R})$ is such that $\left.f(0)=0, f^{\prime}(0) \in\right] \lambda_{i}, \lambda_{i+1}$ [ for some $i \geq 2$ (where $\lambda_{i}$ denotes the $i$-th eigenvalue of $-\Delta$ on $\left.H_{0}^{1}(\Omega)\right)$ and $\lim \sup _{|u| \rightarrow \infty} \frac{f(u)}{u}<\lambda_{1}$ then the above problem has at least four nontrivial solutions, among them, one positive and one negative.

This result has been generalized in several papers. The fact that the last two solutions are sign-changing was proved using Conley index in [9] (it was proved using variational methods related to invariant sets of the minus gradient flow in $\mathcal{C}_{0}^{1}$ in [19]). In [2], in case $\lim _{|u| \rightarrow \infty} f^{\prime}(u)<\lambda_{1}<\lambda_{2}<f^{\prime}(0)$, the authors prove the existence of three nontrivial solutions: one positive, one negative and one sign-changing wedged between the two others. In [8] using Conley index (see also [20] for an argument based on variational methods related to invariant sets of 
the minus gradient flow in $\mathcal{C}_{0}^{1}$ ), the assumption in 0 was generalized considering the situation $\lim _{u \rightarrow 0^{+}} \frac{f(u)}{u}=a$ and $\lim _{u \rightarrow 0^{-}} \frac{f(u)}{u}=b$, with $(a, b)$ above the D'Aujourd'hui curve $\Gamma$ and not on the Fučík spectrum. At last, the restriction $f \in \mathcal{C}^{1}(\mathbb{R})$, made by Höfer in order to obtain the fourth solution, was weakened to $f$ locally Lipschitz in [23].

In the recent paper [26] the authors consider a new direction of generalization. They prove that if $\Omega \subset \mathbb{R}^{N}$ is a bounded smooth domain and $f \in \mathcal{C}^{1}(\bar{\Omega} \times \mathbb{R})$ is such that

(i) the origin is a solution around which the nonlinearity has a slope between two consecutive eigenvalues of order $i \geq 2$, i.e.

$$
f(x, 0)=0 \quad \text { and } \quad \lambda_{i}<f_{u}^{\prime}(x, 0)<\lambda_{i+1} \quad \text { for some } i \geq 2 ;
$$

(ii) in a neighbourhood of $+\infty$, the slope $f(x, u) / u$ is smaller than the first eigenvalue $\lambda_{1}$ i.e.

$$
\limsup _{u \rightarrow+\infty} f(x, u) / u<\lambda_{1} \text { uniformly in } x ;
$$

(iii) in a neighbourhood of $-\infty$, the slope $f(x, u) / u$ is bounded and strictly above the first eigenvalue i.e.

$$
\lambda_{1}<\liminf _{u \rightarrow-\infty} f(x, u) / u \leq \limsup _{u \rightarrow-\infty} f(x, u) / u<+\infty \text { uniformly in } x,
$$

then the problem

$$
\begin{array}{cc}
-\Delta u=f(x, u), & \text { in } \Omega, \\
u=0, & \text { on } \partial \Omega,
\end{array}
$$

has one positive solution and two sign-changing solutions. They also suggest that condition (iii) seems technical and ask if it could be dropped. This is the question we are interested in and that we solve partially in this paper.

In Theorems 4.1 and 4.2 we replace the condition (iii) by requiring that

$$
-\infty<\liminf _{u \rightarrow-\infty} f(x, u) / u \leq \limsup _{u \rightarrow-\infty} f(x, u) / u<+\infty \text { uniformly in } x .
$$

In other words, we again require $f$ to be asymptotically linear at $-\infty$ but without restriction on the coefficient.

In Theorem 4.4 we consider the superlinear case for $u \leq 0$. This is done in case $\lambda_{1}$ is, in some appropriate sense, an asymptotically lower bound (not strict) for $f(x, u) / u$ if $u \rightarrow-\infty$. There are mainly three methods to treat the superlinear problems: the method of Rellich-Pohozaev identities and moving planes as introduced in [14], the scaling or blow-up method introduced in [15] and the method of Hardy-Sobolev inequalities as introduced by Brezis and Turner in [3]. An advantage of this third method is that, unlike the other two, it requires only upper bounds on the growth of the nonlinearity and allows $f$ to depend on $x$. As a disadvantage, it does not provide optimal results in terms of growth rate on $f$. Here we use the ideas of this third method, but without requiring $\lambda_{1}<$ $\liminf \operatorname{in}_{u \rightarrow-\infty} f(x, u) / u$. Hence, from a technical point of view, we do not have an 
a priori bound on the part of the solution in the first eigenspace which gives some difficulties.

In Theorem 4.5 we consider the ordinary differential case and require only a lower bound on $f(t, u) / u$ for $u \rightarrow-\infty$ instead of $($ iii $)$.

Finally in Theorems 4.3 and 4.6 , we consider the case where $f$ depends only on $u$. In that case, the lower bound in (iii) is not required. This implies that for the ordinary autonomous case, condition (iii) is not needed at all. An open question is to know whether such a result still holds for the nonautonomous elliptic case.

In all these results we do not attempt to have the best conditions. Our purpose is mainly to exemplify the variational methods related to invariant sets of the minus gradient flow in $\mathcal{C}_{0}^{1}$. These sets arise naturally in relation with lower and upper solutions. Development of this method is one of the main interest of this paper.

The idea to combine variational methods with invariant sets of the minus gradient flow in $\mathcal{C}_{0}^{1}$ seems to go back to Chang [4-6]. Under the existence of wellordered strict lower and upper solutions $\alpha$ and $\beta$, he proves the existence of a local minimum of the functional and localize it in $\operatorname{int}_{\mathcal{C}_{0}^{1}}\left\{u \in \mathcal{C}_{0}^{1}(\Omega) \mid \alpha \leq u \leq \beta\right\}$. In [19], the authors prove a mountain pass theorem in order intervals in a situation related to the Amann's three solutions theorem and obtain the localization of the critical point of mountain pass type. In [24,25], the authors gives conditions in order to get critical points in the border of the attractive set of invariant sets for the minus gradient flow in $\mathcal{C}_{0}^{1}$. In [11], for an O.D.E., the authors consider the minus gradient flow in $\mathcal{C}_{0}^{1}$ in the non-well ordered lower and upper solutions case and obtain the existence and localization of a solution. All these results define the flow in $\mathcal{C}_{0}^{1}$. This is justified by that fact that sets such as $\{u \in X \mid u \leq \beta\}$ and $\{u \in X \mid u \geq \alpha\}$, where $\alpha$ and $\beta$ are lower and upper solutions that can satisfy the boundary conditions, may have empty interior in the $H_{0}^{1}$-topology. This creates major difficulties. An alternative approach, defining the flow in $H_{0}^{1}$, has been used by Conti et al. [7]. Here we use this point of view of [11].

In Sect. 2, we recall some well known facts about the maximum principle and the first eigenfunction, and give several definitions. Section 3 is devoted to the lower and upper solutions method. After some definitions, we recall the existence result in case the lower and upper solutions are well ordered. This result is well known in other contexts. Then we develop our variational method in the case where the lower and upper solutions are not well ordered. The results of this section are direct extensions of corresponding ones developed in [11] for ordinary differential equations. Finally in Sect. 4, we prove our multiplicity results by applying the theory developed in Sect. 3 .

\section{Preliminaries}

Theorem 2.1 (Strong Maximum Principle). Let $\Omega_{1}$ be a bounded domain in $\mathbb{R}^{N}$ with $\partial \Omega_{1}$ of class $\mathcal{C}^{1,1}, p>N$ and $\lambda \geq 0$. If $u \in W^{2, p}\left(\Omega_{1}\right)$ satisfies 


$$
-\Delta u+\lambda u \geq 0, \quad \text { in } \Omega_{1},
$$

then $u$ cannot achieve a minimum $m \leq 0$ in $\Omega_{1}$ unless $u$ is constant.

Proof. [30, Theorem 3.27].

Theorem 2.2 (Hopf Boundary Point Lemma). Let $\Omega_{1}$ be a bounded domain in $\mathbb{R}^{N}$ with $\partial \Omega_{1}$ of class $\mathcal{C}^{1,1}, p>N, \lambda \geq 0, x_{0} \in \partial \Omega_{1}$ and $b_{0}$ such that $\left(b_{0} \mid \nu\left(x_{0}\right)\right)>0$ for $\nu\left(x_{0}\right)$ the outward normal at $x_{0}$. If $u \in W^{2, p}\left(\Omega_{1}\right)$ satisfies $-\Delta u+\lambda u \geq 0$ in $\Omega_{1}$ and $u$ achieves a strict local minimum $m \leq 0$ at $x_{0}$, then

$$
\left(b_{0} \mid \nabla u\left(x_{0}\right)\right)<0 .
$$

Proof. [30, Lemma 3.26].

Let $\varphi_{1}$ denote the first eigenfunction of $-\Delta$ on $H_{0}^{1}(\Omega)$. We know that $\varphi_{1}>0$ in $\Omega$ and $\partial_{\nu} \varphi_{1}<0$ on $\partial \Omega$.

Definition 2.1. Given functions $u, v: \bar{\Omega} \rightarrow \mathbb{R}$, we write

- $u \leq v$ if for all $x \in \bar{\Omega}, u(x) \leq v(x)$;

- $u<v$ if $u \leq v$ and $u \neq v$;

- $u \ll v$ if there exists $\epsilon>0$ such that $u+\epsilon \varphi_{1} \leq v$.

Remark 2.1. Defining in $\mathcal{C}_{0}^{1}(\bar{\Omega})$, the order cone $K=\left\{v \in \mathcal{C}_{0}^{1}(\bar{\Omega}) \mid v \geq 0\right\}$, we observe that $u \ll v$ if and only if $v-u \in \operatorname{int}(K)$.

A simple consequence of Theorems 2.1 and 2.2 is the following corollary.

Corollary 2.3. Let $\Omega$ be a bounded domain in $\mathbb{R}^{N}, p>N$ and $\lambda \geq 0$. If $u \in$ $W^{2, p}(\Omega)$ satisfies

$$
\begin{array}{cc}
-\Delta u+\lambda u \geq 0, & \text { in } \Omega, \\
u \geq 0, & \text { on } \partial \Omega,
\end{array}
$$

then either $u \equiv 0$ in $\bar{\Omega}$ or $u \gg 0$.

Definition 2.2. We say that a function $f: \Omega \times \mathbb{R} \rightarrow \mathbb{R}$ is an $L^{p}$-Carathéodory function if

- for every $u \in \mathbb{R}, f(\cdot, u)$ is measurable on $\Omega$;

- for a.e. $x \in \Omega, f(x, \cdot)$ is continuous on $\mathbb{R}$;

- for each $\rho>0$, there exists $\gamma \in L^{p}(\Omega)$ such that, for all $(x, u) \in \Omega \times[-\rho, \rho]$,

$$
|f(x, u)| \leq \gamma(x) \text {. }
$$

\section{Lower and upper solutions}

\subsection{Definitions}

We define lower and upper solutions in the following way.

Definition 3.1. A function $\alpha \in W^{2, p}(\Omega)$ is a lower solution of (1.1) if 
(i) for a.e. $x \in \Omega,-\Delta \alpha(x) \leq f(x, \alpha(x))$;

(ii) for all $x \in \partial \Omega, \alpha(x) \leq 0$.

Similarly, a function $\beta \in W^{2, p}(\Omega)$ is an upper solution of (1.1) if

(i) for a.e. $x \in \Omega,-\Delta \beta(x) \geq f(x, \beta(x))$;

(ii) for all $x \in \partial \Omega, \beta(x) \geq 0$.

Definition 3.2. A lower solution (resp. an upper solution) of (1.1) is said proper if it is not a solution.

A lower solution $\alpha$ of (1.1) is said strict if, for all $u$ solution of (1.1) with $u \geq \alpha$, we have $u \gg \alpha$.

In a similar way, an upper solution $\beta$ of (1.1) is said strict if, for all $u$ solution of (1.1) with $u \leq \beta$, we have $u \ll \beta$.

Proposition 3.1. Let $f: \Omega \times \mathbb{R}$ be an $L^{p}$-Carathéodory function such that for some $k \geq 0$, for a.e. $x \in \Omega$ and all $u_{1}, u_{2} \in \mathbb{R}$ with $u_{2} \geq u_{1}$,

$$
f\left(x, u_{2}\right)-f\left(x, u_{1}\right) \geq-k\left(u_{2}-u_{1}\right) .
$$

Let $\alpha$ (resp. $\beta$ ) be a proper lower (resp. upper) solution of (1.1). Then $\alpha$ (resp. $\beta$ ) is a strict lower (resp. upper) solution of (1.1).

Proof. Let $u$ be a solution of (1.1) such that $u \geq \alpha$ and define $w=u-\alpha$. Observe that $w$ satisfies

$$
\begin{array}{cc}
-\Delta w+k w \geq f(x, u(x))-f(x, \alpha(x))+k(u(x)-\alpha(x)) \geq 0, & \text { in } \Omega, \\
w \geq 0, & \text { on } \partial \Omega .
\end{array}
$$

Hence we deduce from Corollary 2.3 that $w \gg 0$ which means that $\alpha$ is strict.

\subsection{Well ordered lower and upper solutions}

Theorem 3.2. Let $\alpha_{i} \in W^{2, p}(\Omega)(i=1, \ldots, n)$ and $\beta_{j} \in W^{2, p}(\Omega)(j=1, \ldots, m)$ be respectively lower and upper solutions of (1.1). Assume

$$
\alpha=\max _{i=1, \ldots, n} \alpha_{i} \leq \min _{j=1, \ldots, m} \beta_{j}=\beta .
$$

Define $E=\left\{(x, u) \in \Omega \times \mathbb{R} \mid \min _{i=1, \ldots, n} \alpha_{i} \leq u \leq \max _{j=1, \ldots, m} \beta_{j}\right\}$. Assume $f$ : $E \rightarrow \mathbb{R}$ satisfies $L^{p}$-Carathéodory conditions with $p>N$. Then the problem (1.1) has a minimum solution $u_{\min } \in W^{2, p}(\Omega)$ and a maximum solution $u_{\max } \in W^{2, p}(\Omega)$ in $[\alpha, \beta]$ i.e.

$$
\alpha \leq u_{\min } \leq u_{\max } \leq \beta
$$

and any other solution $u$ of (1.1) such that $\alpha \leq u \leq \beta$ satisfies

$$
u_{\min } \leq u \leq u_{\max } .
$$

Further if $\alpha$ and $\beta$ are strict then for $R>0$ large enough

$$
\operatorname{deg}(I-S, \mathcal{U})=1,
$$

where

$$
\mathcal{U}=\left\{u \in \mathcal{C}_{0}^{1}(\bar{\Omega}) \mid \alpha \ll u \ll \beta,\|u\|_{\mathcal{C}_{0}^{1}}<R\right\}
$$


and $S: \mathcal{C}_{0}^{1}(\bar{\Omega}) \rightarrow \mathcal{C}_{0}^{1}(\bar{\Omega})$ is defined by $S u=v$ where $v \in W^{2, p}(\Omega)$ is the unique solution of

$$
\begin{array}{cc}
-\Delta v=f(x, u), & \text { in } \Omega, \\
v=0, & \text { on } \partial \Omega .
\end{array}
$$

Proof. This can be proved using ideas developed in [12] in order to adapt to the elliptic case the results of [11, Theorem I-3.2, I-2.4, III-2.8].

Remark 3.1. In case $N=1$, we can work with $L^{1}$-Carathéodory functions. See [11] for details.

\subsection{Non well ordered lower and upper solutions}

3.3.1. The minus gradient flow. We shall define the minus gradient flow using the following assumptions:

(H) (i) for some $p>N, f: \Omega \times \mathbb{R} \rightarrow \mathbb{R}:(x, u) \mapsto f(x, u)$ is an $L^{p}$-Carathéodory function, locally Lipschitz in $u$ uniformly in $x$;

(ii) there exists $m \in \mathbb{R}^{+}$such that $f(x, u)+m u$ is increasing in $u$;

(iii) if $N>2$ (resp. $N=2$ ), there exist $a>0$ and $\gamma \in] 1, \frac{N+2}{N-2}[$ (resp. $\gamma \in] 1,+\infty\left[\right.$ ) and $d \in L^{p}(\Omega)$ such that for all $(x, u) \in \Omega \times \mathbb{R}$ we have

$$
|f(x, u)| \leq a|u|^{\gamma}+d(x) .
$$

Remark 3.2. In case $N=1$, we can work with $L^{1}$-Carathéodory functions locally Lipschitz in $u$ uniformly in $x$, satisfying (ii). See [11] for details.

Now, let us define on $H_{0}^{1}(\Omega)$ the scalar product

$$
(u, v)_{H_{0}^{1}}=\int_{\Omega}[\nabla u(x) \nabla v(x)+m u(x) v(x)] d x
$$

and let

$$
F(x, u)=\int_{0}^{u} f(x, s) d s .
$$

It is then easy to see that the functional

$$
\phi: H_{0}^{1}(\Omega) \rightarrow \mathbb{R}, \quad u \mapsto \int_{\Omega}\left[\frac{|\nabla u(x)|^{2}}{2}-F(x, u(x))\right] d x
$$

is of class $\mathcal{C}^{1}$ and

$$
\nabla \phi(u)=u-A(u)
$$

where

$$
A: H_{0}^{1}(\Omega) \rightarrow H_{0}^{1}(\Omega), \quad u \mapsto A u
$$

and $v:=A u$ is defined to be the unique solution of

$$
\begin{array}{cc}
-\Delta v+m v=f(x, u)+m u, & \text { in } \Omega, \\
v=0, & \text { on } \partial \Omega .
\end{array}
$$


Let us notice at last that if Assumptions (H) are satisfied, the function

$$
\nabla \phi: \mathcal{C}_{0}^{1}(\bar{\Omega}) \rightarrow \mathcal{C}_{0}^{1}(\bar{\Omega}),
$$

defined from (3.3) is locally lipschitzian. Next, we define a $\mathcal{C}^{1}$-function $\psi_{r}: \mathbb{R} \rightarrow$ $[0,1]$ such that $\psi_{r}(s)=1$ if $s \geq r$ and $\psi_{r}(s)=0$ if $s \leq r-1$.

We consider then the Cauchy problem

$$
\begin{aligned}
\frac{d}{d t} u & =-\psi_{r}(\phi(u)) \nabla \phi(u)=-\psi_{r}(\phi(u))(u-A(u)), \\
u(0) & =u_{0},
\end{aligned}
$$

where $u_{0} \in \mathcal{C}_{0}^{1}(\bar{\Omega})$. From the theory of ordinary differential equations, we know that the solution $u\left(\cdot ; u_{0}\right)$ of $(3.5)$ exists, is unique, and is defined in the future on a maximal interval $\left[0, \omega\left(u_{0}\right)\left[\right.\right.$. We also know that for any $t \in\left[0, \omega\left(u_{0}\right)[\right.$, the function $u(t ; \cdot): \mathcal{C}_{0}^{1}(\bar{\Omega}) \rightarrow \mathcal{C}_{0}^{1}(\bar{\Omega})$ is continuous. We call the minus gradient flow the local dynamical system defined on $\mathcal{C}_{0}^{1}(\bar{\Omega})$ by $u\left(t ; u_{0}\right)$.

A first result shows that the solutions of (3.5) are defined for all $t \geq 0$.

Proposition 3.3. Let Assumptions $(H)$ be satisfied and $u\left(t ; u_{0}\right)$ be the minus gradient flow defined for some $r \in \mathbb{R}$. Then for any $u_{0} \in \mathcal{C}_{0}^{1}(\bar{\Omega})$ we have $\omega\left(u_{0}\right)=+\infty$.

Proof. The proof follows the line of [11, Proposition IV-3.1] together with a bootstrap argument that can be found for example in [21].

3.3.2. Invariant sets. An important property of the cones

$$
C_{\alpha}=\left\{u \in \mathcal{C}_{0}^{1}(\bar{\Omega}) \mid u \gg \alpha\right\}
$$

and

$$
C^{\beta}=\left\{u \in \mathcal{C}_{0}^{1}(\bar{\Omega}) \mid u \ll \beta\right\},
$$

which are associated to lower and upper solutions $\alpha$ and $\beta$ of (1.1) is that they are positively invariant. To make this precise, let us introduce the following definitions.

Definition 3.3. Let $u\left(t ; u_{0}\right)$ be the minus gradient flow defined for some $r \in \mathbb{R}$. A nonempty set $M \subset \mathcal{C}_{0}^{1}(\bar{\Omega})$ is called a positively invariant set if

$$
\forall u_{0} \in M, \forall t \geq 0, \quad u\left(t ; u_{0}\right) \in M .
$$

As a first example, notice that the set

$$
\phi^{c}=\left\{u \in \mathcal{C}_{0}^{1}(\bar{\Omega}) \mid \phi(u)<c\right\}
$$

is positively invariant. Also, unions and intersections of positively invariant sets are positively invariant.

Proposition 3.4. Let Assumptions $(H)$ be satisfied and $u\left(t ; u_{0}\right)$ be the minus gradient flow defined for some $r \in \mathbb{R}$.

Assume $\alpha \in W^{2, p}(\Omega)$ is a lower solution of (1.1). Then the set $C_{\alpha}$ defined from (3.6) is positively invariant. 
Similarly, if $\beta \in W^{2, p}(\Omega)$ is an upper solution of (1.1), the set $C^{\beta}$ defined from (3.7) is positively invariant.

Proof. See [11, Proposition IV-3.3].

3.3.3. Non well-ordered lower and upper solutions. The first result of this section provides Palais-Smale type sequences from non well-ordered lower and upper solutions. As usual, this proposition gives a solution of (1.1) with the help of the Palais-Smale condition.

In order to obtain existence of solutions of (1.1), we need to prove that the sequence $\left(u\left(t_{n} ; u_{0}\right)\right)_{n}$ converges toward such a solution, which holds true in case we assume the Palais-Smale Condition (PS).

Recall that the functional $\phi: X \rightarrow \mathbb{R}$ satisfies the Palais-Smale Condition $(P S)$ if for every sequence $\left(u_{n}\right)_{n} \subset X$ such that $\phi\left(u_{n}\right)$ is bounded and $\nabla \phi\left(u_{n}\right) \rightarrow 0$, there exists a subsequence that converges to some function $u \in X$.

Theorem 3.5. Let Assumptions $(H)$ be satisfied. Suppose that $\alpha$ and $\beta \in W^{2, p}(\Omega)$ are lower and upper solutions of (1.1) with $\alpha \not \leq \beta$. Define $C_{\alpha}$ and $C^{\beta}$ from (3.6) and (3.7),

$$
\begin{gathered}
\Gamma=\left\{\gamma \in \mathcal{C}\left([0,1], \mathcal{C}_{0}^{1}(\bar{\Omega})\right) \mid \gamma(0) \in C^{\beta}, \gamma(1) \in C_{\alpha}\right\}, \\
T_{\gamma}=\left\{s \in[0,1] \mid \gamma(s) \in \mathcal{C}_{0}^{1}(\bar{\Omega}) \backslash\left(C^{\beta} \cup C_{\alpha}\right)\right\}
\end{gathered}
$$

and assume

$$
c:=\inf _{\gamma \in \Gamma} \max _{s \in T_{\gamma}} \phi(\gamma(s)) \in \mathbb{R},
$$

where $\phi(u)$ is defined from (3.2). At last, let $u\left(t ; u_{0}\right)$ be the minus gradient flow defined with $r=c-1$.

Then, for any $\delta \in] 0,1\left[\right.$, there exists $u_{0} \in \mathcal{C}_{0}^{1}(\bar{\Omega})$ such that

$$
\forall t>0, \quad u\left(t ; u_{0}\right) \in \phi^{-1}([c-\delta, c+\delta]) \backslash\left(C^{\beta} \cup C_{\alpha}\right)
$$

and there exists an increasing unbounded sequence $\left(t_{n}\right)_{n} \subset \mathbb{R}^{+}$such that

$$
\nabla \phi\left(u\left(t_{n} ; u_{0}\right)\right) \stackrel{H_{0}^{1}}{\rightarrow} 0 \quad \text { as } n \rightarrow \infty .
$$

If moreover the Palais-Smale Condition (PS) is satisfied, then there exists $v \in \mathcal{C}_{0}^{1}(\bar{\Omega}) \backslash\left(C^{\beta} \cup C_{\alpha}\right)$ solution of $(1.1)$ such that $\phi(v)=c$.

Proof. The first part of the result can be proved as in [11, Proposition IV-3.4]. The second part is proved following the ideas of [11, Theorem IV-3.5] together with a bootstrap argument that can be found for example in [21].

\section{Multiplicity results}

In this section we will apply the previous results on a modified problem. Hence we can assume weaker condition than $(\mathrm{H})$. 
$\left(H^{\prime}\right) \quad$ Let $p>N$ and $f: \Omega \times \mathbb{R} \rightarrow \mathbb{R}:(x, u) \mapsto f(x, u)$ be an $L^{p}$-Carathéodory function, locally $L^{\infty}$-Lipschitz in $u$ uniformly in $x$.

Remark 4.1. As in the previous section, in case $N=1$, we can work with $L^{1}$ Carathéodory functions locally Lipschitz in $u$ uniformly in $x$.

We denote by $\lambda_{k}$ the $k$-th eigenvalue of $-\Delta$ on $H_{0}^{1}(\Omega)$ and $\varphi_{k}$ the corresponding eigenfunction with $\left\|\varphi_{k}\right\|_{\infty}=1$.

Theorem 4.1. Let Assumptions $\left(H^{\prime}\right)$ be satisfied and assume

(i) there exist $\lambda>\lambda_{2}$ and $\delta>0$ such that for a.e. $x \in \Omega$ and all $u \in[-\delta, \delta] \backslash\{0\}$,

$$
\frac{f(x, u)}{u} \geq \lambda
$$

(ii) there exist $\mu<\lambda_{1}$ and $R>0$ such that for a.e. $x \in \Omega$ and all $u \geq R$,

$$
\frac{f(x, u)}{u} \leq \mu
$$

(iii) there exist $a<0<b$ and $R^{\prime}>0$ such that for a.e. $x \in \Omega$ and all $u \leq-R^{\prime}$,

$$
a \leq \frac{f(x, u)}{u} \leq b .
$$

Then the problem (1.1) has at least two nontrivial solutions: one positive and the other one sign-changing.

Proof. Step 1 - There exists $\beta_{1} \gg 0$ which is a proper upper solution of (1.1). Let $h \in L^{p}(\Omega)$ be such that $h \geq 0$ and for a.e. $x \in \Omega$ and all $u \geq 0$,

$$
f(x, u)<\mu u+h(x) .
$$

Define then $\beta_{1}$ to be the solution of

$$
\begin{array}{cc}
-\Delta u=\mu u+h(x), & \text { in } \Omega, \\
u=0, & \text { on } \partial \Omega .
\end{array}
$$

As $\mu<\lambda_{1}$ and $h \geq 0$ we have from the maximum principle and the KreinRutmann theorem that $\beta_{1} \gg 0$ and

$$
\begin{array}{cc}
-\Delta \beta_{1}(x)=\mu \beta_{1}(x)+h(x)>f\left(x, \beta_{1}(x)\right), & \text { in } \Omega, \\
\beta_{1}=0, & \text { on } \partial \Omega,
\end{array}
$$

i.e. $\beta_{1} \gg 0$ is a proper upper solution.

Step 2-For every nontrivial nonnegative solution $u$ of (1.1) we have $u \geq \delta \varphi_{1}$. Let $u^{*}$ be a nontrivial nonnegative solution of (1.1) and define $\eta=\max \{\tau \geq 0 \mid$ $\left.u^{*}-\tau \varphi_{1} \geq 0\right\}$.

Observe first that, as $f$ is locally $L^{\infty}$-Lipschitz in $u$ uniformly in $x$, we have $k>0$ such that

$$
\begin{array}{cc}
-\Delta u^{*}+k u^{*}=f\left(x, u^{*}\right)+k u^{*} \geq f(x, 0)=0, & \text { in } \Omega, \\
u^{*}=0, & \text { on } \partial \Omega .
\end{array}
$$

Hence, by Corollary 2.3, $u^{*} \gg 0$ and $\eta>0$.

Assume now that $\eta<\delta$. By definition of $\eta$, two cases may occur. 
If there exists $x_{0} \in \Omega$ such that $u^{*}\left(x_{0}\right)-\eta \varphi_{1}\left(x_{0}\right)=0$ then there exists $\Omega_{1} \subset \Omega$ such that $x_{0} \in \Omega_{1}, u^{*}(x) \leq \delta$ on $\Omega_{1}$ and a.e. in $\Omega_{1}$

$$
\begin{aligned}
-\Delta\left(u^{*}-\eta \varphi_{1}\right)(x)=f\left(x, u^{*}(x)\right)-\eta \lambda_{1} \varphi_{1}(x) & \geq \lambda u^{*}(x)-\eta \lambda_{1} \varphi_{1}(x) \\
& >\lambda_{1}\left(u^{*}-\eta \varphi_{1}\right)(x) \geq 0 .
\end{aligned}
$$

By the maximum principle $u^{*}-\eta \varphi_{1}=0$ on $\Omega_{1}$ which contradicts the above strict inequality.

If not, by definition of $\eta$, there exists $x_{0} \in \partial \Omega$ with $\partial_{\nu}\left(u^{*}-\eta \varphi_{1}\right)\left(x_{0}\right)=0$ and there exists $\Omega_{1} \subset \Omega$ such that $u^{*} \leq \delta$ on $\Omega_{1}$. As above we have $-\Delta\left(u^{*}-\eta \varphi_{1}\right)>0$ on $\Omega_{1}$ which contradicts the Hopf maximum principle.

Step 3-For every nontrivial nonpositive solution $u$ of (1.1) we have $u \leq-\delta \varphi_{1}$. The proof is similar to the one of Step 2.

Step 4-Existence of proper lower and upper solutions $\alpha_{1}$ and $\beta_{2}$ with $-\delta \varphi_{1} \leq$ $\beta_{2} \ll 0 \ll \alpha_{1} \leq \delta \varphi_{1}$. Fix $\epsilon>0$ small enough so that $\epsilon<\min \left\{\delta / 4, \lambda-\lambda_{2}\right\}$ and $4 \epsilon \varphi_{1} \ll \beta_{1}$. It is easy to see that $\beta_{2}=-\epsilon \varphi_{1}$ and $\alpha_{1}=\epsilon \varphi_{1}$ are respectively proper upper and lower solutions of (1.1) since

$$
\begin{gathered}
-\Delta \beta_{2}(x)=-\epsilon \lambda_{1} \varphi_{1}(x)>f\left(x,-\epsilon \varphi_{1}(x)\right)=f\left(x, \beta_{2}(x)\right), \\
-\Delta \alpha_{1}(x)=\epsilon \lambda_{1} \varphi_{1}(x)<f\left(x, \epsilon \varphi_{1}(x)\right)=f\left(x, \alpha_{1}(x)\right) .
\end{gathered}
$$

Step 5-Existence of a positive solution By construction we have lower and upper solutions $\alpha_{1}$ and $\beta_{1}$ of (1.1) with $\alpha_{1} \leq \beta_{1}$. Hence, by Theorem 3.2 there is a solution $u_{1}$ of (1.1) with $\alpha_{1} \leq u_{1} \leq \beta_{1}$. As $\alpha_{1} \gg 0$ we have $u_{1} \gg 0$.

Step 6-The modified problem. Consider the modified problem

$$
\begin{array}{cc}
-\Delta u=f_{r}(x, u), & \text { in } \Omega, \\
u=0, & \text { on } \partial \Omega,
\end{array}
$$

where

$$
\begin{aligned}
f_{r}(x, u) & =0, & & \text { if } u<-r-1, \\
& =(1+r+u) f(x, u), & & \text { if }-r-1 \leq u<-r, \\
& =f(x, u), & & \text { if }-r \leq u \leq \beta_{1}(x), \\
& =f\left(x, \beta_{1}(x)\right), & & \text { if } u>\beta_{1}(x) .
\end{aligned}
$$

Observe that, by the maximum principle as in Theorem 3.2, every solution $u$ of (4.9) satisfies $u \leq \beta_{1}$.

Claim: There exists $K>0$ such that for all $r>K$ and all solutions $u$ of (4.9) with $u \notin C^{\beta_{2}} \cup C_{\alpha_{1}}$ we have $\|u\|_{\mathcal{C}^{1}}<K$. By assumption (H') and (iii), there exists $\gamma \in L^{p}(\Omega)$ such that, for all $r$

$$
f_{r}(x, u)=-g_{r}(x, u) u^{-}+h_{r}(x, u),
$$

with

$$
\begin{gathered}
a \leq g_{r}(x, u) \leq b \\
\left|h_{r}(x, u)\right| \leq \gamma(x)
\end{gathered}
$$


Assume by contradiction there exist sequences $\left(r_{n}\right)_{n}$ and $\left(u_{n}\right)_{n} \notin C^{\beta_{2}} \cup C_{\alpha_{1}}$, where $r_{n} \geq n$ and $u_{n}$ is a solution of (4.9) with $r=r_{n}$ such that $\left\|u_{n}\right\|_{\mathcal{C}^{1}} \geq n$.

Consider now the functions $v_{n}=u_{n} /\left\|u_{n}\right\|_{\mathcal{C}^{1}}$ which solve the problems

$$
\begin{array}{cc}
-\Delta v_{n}=-g_{r_{n}}\left(x, u_{n}\right) v_{n}^{-}+\frac{h_{r_{n}}\left(x, u_{n}\right)}{\left\|u_{n}\right\|_{\mathcal{C}^{1}}}, & \text { in } \Omega, \\
v_{n}=0, & \text { on } \partial \Omega .
\end{array}
$$

Going to subsequence, we can assume that

$$
g_{r_{n}}\left(\cdot, u_{n}\right) \rightarrow q \text { in } L^{p}(\Omega),
$$

with $a \leq q \leq b$,

$$
\frac{h_{r_{n}}\left(\cdot, u_{n}\right)}{\left\|u_{n}\right\|_{\mathcal{C}^{1}}} \rightarrow 0 \text { in } L^{p}(\Omega),
$$

$v_{n}$ is bounded in $W^{2, p}(\Omega)$ and hence

$$
v_{n} \rightarrow v \text { in } W^{2, p}(\Omega) \quad \text { and } \quad v_{n} \rightarrow v \text { in } \mathcal{C}_{0}^{1}(\bar{\Omega}) \text { with }\|v\|_{\mathcal{C}^{1}}=1 .
$$

As $u_{n} \leq \beta_{1}$, we deduce that $v \leq 0$ is a solution of

$$
\begin{gathered}
-\Delta v=-q v^{-}, \quad \text { in } \Omega, \\
v=0, \quad \text { on } \partial \Omega .
\end{gathered}
$$

Hence we have

$$
\begin{array}{cc}
-\Delta v-a v=(q-a) v \leq 0, & \text { in } \Omega, \\
v=0, & \text { on } \partial \Omega,
\end{array}
$$

which implies $v \ll 0$ by Corollary 2.3 .

By [16, Lemma 3.1], there exist $C_{1}, C_{2}>0$ such that, for any $n$ large enough, $v_{n} \leq C_{1} v$ and $C_{2} v \ll \beta_{2}$. As $v_{n}=u_{n} /\left\|u_{n}\right\|_{\mathcal{C}^{1}}$ this implies that, for $n$ large enough, $u_{n} \ll \beta_{2}$ which contradict the assumption $u_{n} \notin C^{\beta_{2}} \cup C_{\alpha_{1}}$.

Now let $r>K$ be fixed and consider the modified functional

$$
\phi_{r}(u)=\int_{\Omega}\left[\frac{|\nabla u(x)|^{2}}{2}-F_{r}(x, u(x))\right] d x,
$$

with $F_{r}(x, u)=\int_{0}^{u} f_{r}(x, \xi) d \xi$. Observe that $f_{r}$ satisfies assumption $(\mathrm{H})$ and that, by Proposition 3.1, every proper lower (resp. upper) solution of (4.9) is strict. In particular $\alpha_{1}$ is strict and $u_{1} \gg \alpha_{1}$.

Step 7-Existence of a second nontrivial solution. We apply Theorem 3.5 on $\phi_{r}$ with $\alpha=\alpha_{1}$ and $\beta=\beta_{2}$. Observe that as $\phi_{r}$ is coercive, it satisfies the PalaisSmale Condition and $c \in \mathbb{R}$. This observation proves the existence of a solution $u_{2} \in \mathcal{C}_{0}^{1}(\bar{\Omega}) \backslash\left(C^{\beta_{2}} \cup C_{\alpha_{1}}\right)$, i.e. $u_{2} \neq u_{1}$. The main problem is to see that $u_{2}$ is not the trivial solution. To this aim, we prove that $c=\phi_{r}\left(u_{2}\right)<0=\phi_{r}(0)$.

Define $\Gamma$ from (3.8), with $\alpha=\alpha_{1}, \beta=\beta_{2}$, and $\gamma \in \Gamma$ by

$$
\gamma(s)=2 \epsilon\left[(2 s-1) \varphi_{1}+(1-|2 s-1|) \varphi_{2}\right] .
$$

Observe that

$$
\begin{gathered}
\gamma(0)=-2 \epsilon \varphi_{1} \ll \beta_{2}, \quad \gamma(1)=2 \epsilon \varphi_{1} \gg \alpha_{1}, \\
-4 \varepsilon \varphi_{1} \leq \gamma(s) \leq 4 \varepsilon \varphi_{1} \ll \beta_{1} \quad \text { for all } s \in[0,1] .
\end{gathered}
$$


Moreover we have

$$
\begin{aligned}
\phi_{r}(\gamma(s))= & \int_{\Omega}\left[2 \epsilon^{2}\left[(2 s-1)^{2} \nabla \varphi_{1}^{2}(x)+(1-|2 s-1|)^{2} \nabla \varphi_{2}^{2}(x)\right]\right. \\
& \left.-F_{r}\left(x, 2 \epsilon\left[(2 s-1) \varphi_{1}(x)+(1-|2 s-1|) \varphi_{2}(x)\right]\right)\right] d x \\
\leq & \int_{\Omega}\left[2 \epsilon^{2}\left[(2 s-1)^{2} \lambda_{1} \varphi_{1}^{2}(x)+(1-|2 s-1|)^{2} \lambda_{2} \varphi_{2}^{2}(x)\right]\right. \\
& \left.-\lambda 2 \epsilon^{2}\left[(2 s-1) \varphi_{1}(x)+(1-|2 s-1|) \varphi_{2}(x)\right]^{2}\right] d x \\
\leq & 2 \epsilon^{2}|\Omega|\left[(2 s-1)^{2}\left(\lambda_{1}-\lambda\right)+(1-|2 s-1|)^{2}\left(\lambda_{2}-\lambda\right)\right] \\
\leq & 2 \epsilon^{2}|\Omega|\left[(2 s-1)^{2}+(1-|2 s-1|)^{2}\right]\left(\lambda_{2}-\lambda\right) \\
\leq & -\epsilon^{3}|\Omega| .
\end{aligned}
$$

Hence, by definition of $c$, we have $c \leq-\epsilon^{3}|\Omega|<0$, which implies the second solution $u_{2}$ is nontrivial.

Step 8 - The function $u_{2}$ changes sign. Assume $u_{2}>0$, then by Step $2 u_{2} \geq \delta \varphi_{1} \geq$ $\alpha_{1}$ which contradicts the localization of $u_{2}$. We prove in a similar way that $u_{2}$ cannot be nonpositive. Therefore $u_{2}$ changes sign.

An additional solution can be obtained combining variational method and degree theory. Here we impose that the slope $\frac{f(x, u)}{u}$ lies between two consecutive eigenvalues for small values of $u$.

Theorem 4.2. Assume that $f \in \mathcal{C}^{1}(\bar{\Omega} \times \mathbb{R})$ satisfies

$\left(i^{\prime}\right)$ there exist $p, q, k \geq 2(k \in \mathbb{N})$ and $\delta>0$ such that for a.e. $x \in \Omega$ and all $u \in[-\delta, \delta] \backslash\{0\}$,

$$
\lambda_{k}<p \leq \frac{f(x, u)}{u} \leq q<\lambda_{k+1}
$$

(ii) there exist $\mu<\lambda_{1}$ and $R>0$ such that for a.e. $x \in \Omega$ and all $u \geq R$,

$$
\frac{f(x, u)}{u} \leq \mu
$$

(iii) there exist $a<0<b$ and $R^{\prime}>0$ such that for a.e. $x \in \Omega$ and all $u \leq-R^{\prime}$,

$$
a \leq \frac{f(x, u)}{u} \leq b .
$$

Then the problem (1.1) has at least three nontrivial solutions $u_{i}$ : one positive and two sign-changing.

Proof. Part 1-Modified problem. As in the proof of Theorem 4.1, we choose a lower solutions $\alpha_{1}$ and upper solutions $\beta_{i}$ such that

$$
-\delta \varphi_{1} \ll \beta_{2}=-\varepsilon \varphi_{1}, \quad \alpha_{1}=\varepsilon \varphi_{1} \ll \delta \varphi_{1} \quad \text { and } \quad \alpha_{1} \ll \beta_{1},
$$

where $\varepsilon \in] 0, \delta[$. Moreover as in that proof we have a constant $K>0$ such that for all $r>K$ and all solutions $u \notin C^{\beta_{2}} \cup C_{\alpha_{1}}$ of (4.9) we have $\|u\|_{\mathcal{C}^{1}}<K$ and moreover $u \leq \beta_{1}$. Hence we consider the problem (4.9) with $r>K$. Observe that 
$\alpha_{2}=-r-2$ is a lower solution of that problem with $\alpha_{2} \ll \beta_{2}$. From Theorem 3.2 , the problem (4.9) has two solutions $u_{2} \leq \beta_{2}$ and $u_{1} \geq \alpha_{1}$ such that $u_{2}$ is the maximum solution in $\left[\alpha_{2}, \beta_{2}\right]$ and $u_{1}$ is the minimum solution in $\left[\alpha_{1}, \beta_{1}\right]$ of (4.9). Moreover we can prove as in the proof of Steps 2 and 3 of Theorem 4.1 that

$$
u_{2} \leq-\delta \varphi_{1} \text { and } u_{1} \geq \delta \varphi_{1} .
$$

Consider now the modified problem

$$
\begin{array}{cc}
-\Delta u=\bar{f}(x, u), & \text { in } \Omega, \\
u=0, & \text { on } \partial \Omega,
\end{array}
$$

where

$$
\begin{aligned}
\bar{f}(x, u) & =f_{r}\left(x, u_{2}(x)\right), & & \text { if } u<u_{2}(x), \\
& =f_{r}(x, u), & & \text { if } u_{2}(x) \leq u<u_{1}(x), \\
& =f_{r}\left(x, u_{1}(x)\right), & & \text { if } u_{1}(x) \leq u
\end{aligned}
$$

and the corresponding functional

$$
\bar{\phi}(u)=\int_{\Omega}\left[\frac{|\nabla u(x)|^{2}}{2}-\bar{F}(x, u(x))\right] d x,
$$

with $\bar{F}(x, u)=\int_{0}^{u} \bar{f}(x, s) d s$. Observe that $\bar{f}$ satisfies conditions $(\mathrm{H})$ with $m$ given by the locally $L^{\infty}$-Lipschitz condition of $f$ on $\left[\min u_{2}, \max u_{1}\right]$. By the maximum principle as in Theorem 3.2, it is easy to see that every solution $u$ of (4.10) satisfies $u_{2} \leq u \leq u_{1}$ and is a solution of (4.9). Moreover, by extremality of $u_{1}$ and $u_{2}$, every solution $u$ of (4.10) but $u_{1}$ and $u_{2}$ satisfies $u \notin C^{\beta_{2}} \cup C_{\alpha_{1}}$ and therefore, by the choice of $r$, is a solution of (1.1).

As in the proof of Theorem 4.1, we see that the problem (4.10) has a third solution $u_{3} \neq 0$, which changes sign and is such that

$$
\bar{\phi}\left(u_{3}\right)=\inf _{\gamma \in \Gamma} \max _{s \in T_{\gamma}} \bar{\phi}(\gamma(s))<0,
$$

where $\Gamma$ and $T_{\gamma}$ are defined from (3.8). Notice also that $\bar{\phi}$ satisfies the Palais-Smale Condition.

Part 2-Existence of a further nontrivial solution $u_{4}$. Assume by contradiction that the only solutions of (4.10) are $u_{1}, u_{2}, u_{3}$ and 0 . As $u_{2}-1$ and $\alpha_{1}$ are strict lower solutions of (4.10) and $\beta_{2}$ and $u_{1}+1$ are strict upper solutions of (4.10), we have, by Theorem 3.2,

$$
\operatorname{deg}\left(I-\bar{A}, \Omega_{1}\right)=1 \quad \text { and } \quad \operatorname{deg}\left(I-\bar{A}, \Omega_{2}\right)=1
$$

where, for $R$ large enough,

$$
\begin{aligned}
& \Omega_{1}=\left\{u \in \mathcal{C}_{0}^{1}(\bar{\Omega}) \mid u_{2}-1 \ll u \ll \beta_{2},\|u\|_{\mathcal{C}^{1}}<R\right\}, \\
& \Omega_{2}=\left\{u \in \mathcal{C}_{0}^{1}(\bar{\Omega}) \mid \alpha_{1} \ll u \ll u_{1}+1,\|u\|_{\mathcal{C}^{1}}<R\right\},
\end{aligned}
$$

and $\bar{A}$ is defined from

$$
\bar{A}: \mathcal{C}_{0}^{1} \rightarrow \mathcal{C}_{0}^{1}(\bar{\Omega}), u \mapsto \bar{A} u
$$


where $v:=\bar{A} u$ is the unique solution of

$$
\begin{array}{cc}
-\Delta v=\bar{f}(x, u), & \text { in } \Omega, \\
v=0, & \text { on } \partial \Omega .
\end{array}
$$

Suppose $\bar{\phi}\left(u_{1}\right) \geq \bar{\phi}\left(u_{2}\right)$, a similar argument holds if $\bar{\phi}\left(u_{2}\right)>\bar{\phi}\left(u_{1}\right)$. As the only critical points are $\left\{u_{1}, u_{2}, u_{3}, 0\right\}$, by [13, Theorem 5.10] (see also [11, Theorem A-2.4]), there exists $\gamma>0$ such that

$$
\inf \left\{\bar{\phi}(u) \mid\left\|u-u_{1}\right\|_{H_{0}^{1}}=\gamma\right\}>\bar{\phi}\left(u_{1}\right) .
$$

Next, by [28, Theorem 4.10] and [18] (see also [11, Theorem A-2.3]), there is a critical point of mountain pass type. As $k \geq 2$, it cannot be 0 . Hence this point is $u_{3}$ and from [18] and [1] (see also [6, Corollary 2-3.1] and [11, Theorem A-2.6, Corollary A-2.7]) and [11, Theorem A-1.7], there exists $\varepsilon>0$ such that

$$
\operatorname{deg}\left(I-\bar{A}, B\left(u_{3}, \varepsilon\right)\right)=-1 .
$$

Moreover, as $\bar{A}\left(\mathcal{C}_{0}^{1}(\bar{\Omega})\right) \subset B(0, \bar{R})$ for some $\bar{R}>0$,

$$
\operatorname{deg}(I-\bar{A}, B(0, \bar{R}))=1 .
$$

Let us prove next that, for $r$ small enough,

$$
|\operatorname{deg}(I-\bar{A}, B(0, r))|=1 .
$$

Consider the homotopy

$$
\begin{array}{cc}
-\Delta u=s \bar{f}(x, u)+(1-s) \frac{p+q}{2} u=0, & \text { in } \Omega, \\
u=0, & \text { on } \partial \Omega .
\end{array}
$$

Notice that for a.e. $x \in \bar{\Omega}$ and all $u \in\left[\max \left(-\delta, u_{2}(x)\right), \min \left(u_{1}(x), \delta\right)\right]$

$$
\lambda_{k}<p \leq s \frac{\bar{f}(x, u)}{u}+(1-s) \frac{p+q}{2} \leq q<\lambda_{k+1} .
$$

Any solution $u$ of (4.11) is such that

$$
\begin{array}{cc}
-\Delta u=P(x) u, & \text { in } \Omega, \\
u=0, & \text { on } \partial \Omega .
\end{array}
$$

with

$$
P(x):=s \frac{\bar{f}(x, u(x))}{u(x)}+(1-s) \frac{p+q}{2} .
$$

We can find $r>0$ small enough such that if $u \in \partial B(0, r)$ we have $P(x) \in[p, q] \subset$ ]$\lambda_{k}, \lambda_{k+1}[$ for $x \in \Omega$. By eigenvalue comparison, we conclude that $u \equiv 0$. Hence, using $[27$, p. 66] or $[29$, vol. 2, p. 185] and the homotopy invariance of the degree, we obtain

$$
|\operatorname{deg}(I-\bar{A}, B(0, r))|=1 .
$$

We come now to the contradiction

$$
\begin{aligned}
\operatorname{deg}(I-\bar{A}, B(0, \bar{R}))= & \operatorname{deg}\left(I-\bar{A}, \Omega_{1}\right)+\operatorname{deg}\left(I-\bar{A}, \Omega_{2}\right) \\
& +\operatorname{deg}\left(I-\bar{A}, B\left(u_{3}, \varepsilon\right)\right)+\operatorname{deg}(I-\bar{A}, B(0, r)) \\
= & 1+1-1 \pm 1 \neq 1,
\end{aligned}
$$


which proves the existence of an additional nontrivial solution $u_{4}$ of (4.10). Recall that such a solution lies in $\left[u_{1}, u_{2}\right]$ and from the definition of $u_{1}$ and $u_{2}$, we have $u_{4} \notin C^{\beta_{2}} \cup C_{\alpha_{1}}$ and $u_{4}$ is a solution of (1.1). Arguing as in Theorem 4.1, we prove then that this solution changes sign too.

In case $f$ is independent of $x$, we do not need the lower bound on $f(u) / u$ for $u \leq-R^{\prime}$ in Theorems 4.1 and 4.2 .

Theorem 4.3. Let $f: \mathbb{R} \rightarrow \mathbb{R}: u \mapsto f(u)$ be a locally Lipschitz continuous function. Assume

(i) there exist $\lambda>\lambda_{2}$ and $\delta>0$ such that for all $u \in[-\delta, \delta] \backslash\{0\}$,

$$
\frac{f(u)}{u} \geq \lambda
$$

(ii) there exist $\mu<\lambda_{1}$ and $R>0$ such that for all $u \geq R$,

$$
\frac{f(u)}{u} \leq \mu
$$

(iv) there exist $b>0$ and $R^{\prime}>0$ such that for all $u \leq-R^{\prime}$,

$$
\frac{f(u)}{u} \leq b
$$

Then the problem

$$
\begin{aligned}
-\Delta u & =f(u), \quad \text { in } \Omega, \\
u & =0, \quad \text { on } \partial \Omega,
\end{aligned}
$$

has at least two nontrivial solutions: one positive and the other one sign-changing. If moreover $f \in \mathcal{C}^{1}(\mathbb{R})$ and there exist $p, q, k \geq 2(k \in \mathbb{N})$ and $\delta>0$ such that for all $u \in[-\delta, \delta] \backslash\{0\}$,

$$
\lambda_{k}<p \leq \frac{f(u)}{u} \leq q<\lambda_{k+1},
$$

then the problem (4.12) has a second sign-changing solution.

Proof. As in the proof of Theorem 4.1, we have proper upper solutions $\beta_{i}(i=1,2)$ and a proper lower solution $\alpha_{1}$ such that

$$
\beta_{2} \ll 0 \ll \alpha_{1}<\beta_{1} .
$$

In case there exists $a<0$ such that for $u \leq-R^{\prime}, f(u) / u \geq a$, we are reduced to Theorems 4.1 and 4.2. So assume

$$
\liminf _{u \rightarrow-\infty} \frac{f(u)}{u}=-\infty
$$

This implies the existence of a sequence $R_{n} \rightarrow-\infty$ such that $f\left(R_{n}\right)>0$ and hence, for $n$ large enough, $\alpha_{2}=R_{n}$ is a proper lower solution with $\alpha_{2} \leq \beta_{2}$. 
We then apply the same arguments as in Theorems 4.1 and 4.2 on the modified problem

$$
\begin{array}{cc}
-\Delta u=\bar{f}(x, u), & \text { in } \Omega, \\
u=0, & \text { on } \partial \Omega,
\end{array}
$$

where

$$
\begin{aligned}
\bar{f}(x, u) & =f\left(\alpha_{2}\right), & & \text { if } u<\alpha_{2}, \\
& =f(u), & & \text { if } \alpha_{2} \leq u<\beta_{1}(x), \\
& =f\left(\beta_{1}(x)\right), & & \text { if } \beta_{1}(x) \leq u .
\end{aligned}
$$

We can also consider the case where $f$ is superlinear for $u \leq 0$ if, in some sense, $\lambda_{1}$ is asymptotically a lower bound for $\frac{f(x, u)}{u}$ as $u \rightarrow-\infty$.

Theorem 4.4. Theorems 4.1 and 4.2 are valid if we replace condition (iii) by

(v) there are a constant $\delta \in] 1, \frac{N+1}{N-1}\left[\right.$ and a function $h \in L^{p}(\Omega)$ such that

$$
-|u|^{\delta}-h(x) \leq f(x, u)-\lambda_{1} u \leq h(x),
$$

for a.e. $x \in \Omega$ and all $u \leq 0$.

Proof. As in the proof of Theorem 4.1, we have a proper upper solution $\beta_{1} \gg 0$ and we prove that, for $\epsilon<\min \left\{\delta / 4, \lambda-\lambda_{2}\right\}$ such that $4 \epsilon \varphi_{1} \ll \beta_{1}$, the function $\alpha_{1}=\epsilon \varphi_{1}$ is a proper lower solution and the function $\beta_{2}=-\epsilon \varphi_{1}$ is a proper upper solution. Hence we have a positive solution $\alpha_{1} \leq u_{1} \leq \beta_{1}$. Moreover we prove that every nontrivial nonnegative (resp. nonpositive) solution of (1.1) is such that $u \geq \delta \varphi_{1}$ (resp. $u \leq-\delta \varphi_{1}$ ).

Part 1-First modified problem. Consider the modified problem

$$
\begin{array}{cc}
-\Delta u=f_{r}(x, u), & \text { in } \Omega, \\
u=0, & \text { on } \partial \Omega,
\end{array}
$$

where

$$
\begin{aligned}
f_{r}(x, u) & =\lambda_{1} u, & & \text { if } u<-r-1, \\
& =(1+r+u) f(x, u)-(r+u) \lambda_{1} u, & & \text { if }-r-1 \leq u<-r, \\
& =f(x, u), & & \text { if }-r \leq u \leq \beta_{1}(x), \\
& =f\left(x, \beta_{1}(x)\right), & & \text { if } u>\beta_{1}(x) .
\end{aligned}
$$

Observe that, by the maximum principle as in Theorem 3.2, every solution $u$ of (4.13) satisfies $u \leq \beta_{1}$.

Claim 1: There exists $K_{1}>0$ such that for all $r>K_{1}$ and all solutions $u$ of (4.13) with $u \notin C^{\beta_{2}} \cup C_{\alpha_{1}}$ we have $\|u\|_{\mathcal{C}^{1}}<K_{1}$. Define

$$
\begin{gathered}
g_{r}(x, u)=\min \left\{\max \left\{f_{r}(x, u)-\lambda_{1} u,-|u|^{\delta}\right\}, 0\right\}, \\
h_{r}(x, u)=f_{r}(x, u)-\lambda_{1} u-g_{r}(x, u) .
\end{gathered}
$$


Hence (4.13) becomes

$$
\begin{array}{cc}
-\Delta u=\lambda_{1} u+g_{r}(x, u)+h_{r}(x, u), & \text { in } \Omega, \\
u=0, & \text { on } \partial \Omega,
\end{array}
$$

and there exists $\gamma_{1} \in L^{p}(\Omega)$ such that, for a.e. $x \in \Omega$, all $u \leq \beta_{1}(x)$ and all $r>0$, we have

$$
\begin{gathered}
-|u|^{\delta} \leq g_{r}(x, u) \leq 0, \\
\left|h_{r}(x, u)\right| \leq \gamma_{1}(x) .
\end{gathered}
$$

Assume by contradiction that for each $n$ there exist $r_{n}>n$ and $u_{n}$, solution of (4.14) for $r=r_{n}$, such that $u_{n} \notin C^{\beta_{2}} \cup C_{\alpha_{1}}, u_{n} \leq \beta_{1}$ and $\left\|u_{n}\right\|_{\mathcal{C}^{1}} \geq n$. Let us write $u_{n}=\xi_{n} \varphi_{1}+w_{n}$ where $\int_{\Omega} w_{n} \varphi_{1} d x=0$.

Step 1. There exists $D>0$ such that, for all $n \in \mathbb{N}$,

$$
\left\|w_{n}\right\|_{H_{0}^{1}} \leq D\left(\left|\xi_{n}\right|^{\delta(1-\mu)}+1\right)
$$

where $\mu=\frac{N+2-\delta(N-2)}{2(N+1)-\delta(N-2)}$. Multiplying (4.14) by $\varphi_{1}$ and integrating we obtain

$$
\begin{aligned}
\int_{\Omega}\left|g_{r_{n}}\left(x, u_{n}(x)\right)\right| \varphi_{1}(x) d x & =-\int_{\Omega} g_{r_{n}}\left(x, u_{n}(x)\right) \varphi_{1}(x) d x \\
& =\int_{\Omega} h_{r_{n}}\left(x, u_{n}(x)\right) \varphi_{1}(x) d x \leq \int_{\Omega} \gamma_{1}(x) \varphi_{1}(x) d x=: C_{1} .
\end{aligned}
$$

Multiplying (4.14) by $w_{n}$ and integrating we obtain, using Hölder inequality,

$$
\begin{aligned}
& C_{2}\left\|w_{n}\right\|_{H_{0}^{1}}^{2} \leq \int_{\Omega}\left[\left|\nabla w_{n}\right|^{2}-\lambda_{1} w_{n}^{2}\right] d x=\int_{\Omega}\left[g_{r_{n}}\left(x, u_{n}\right) w_{n}+h_{r_{n}}\left(x, u_{n}\right) w_{n}\right] d x \\
& \leq \int_{\Omega}\left|g_{r_{n}}\left(x, u_{n}\right)\right|^{\mu} \varphi_{1}^{\mu}\left|g_{r_{n}}\left(x, u_{n}\right)\right|^{1-\mu} \frac{\left|w_{n}\right|}{\varphi_{1}^{\mu}} d x+\int_{\Omega} h_{r_{n}}\left(x, u_{n}\right) w_{n} d x \\
& \leq\left(\int_{\Omega}\left|g_{r_{n}}\left(x, u_{n}\right)\right| \varphi_{1} d x\right)^{\mu}\left(\int_{\Omega}\left|g_{r_{n}}\left(x, u_{n}\right)\right| \frac{\left|w_{n}\right|^{\frac{1}{1-\mu}}}{\varphi_{1}^{\frac{\mu}{1-\mu}}} d x\right)^{1-\mu} \\
& +\int_{\Omega} h_{r_{n}}\left(x, u_{n}\right) w_{n} d x \\
& \leq C_{3}\left(\int_{\Omega}\left|u_{n}\right|^{\delta} \frac{\left|w_{n}\right|^{\frac{1}{1-\mu}}}{\varphi_{1}^{\frac{\mu}{1-\mu}}} d x\right)^{1-\mu}+\int_{\Omega} h_{r_{n}}\left(x, u_{n}\right) w_{n} d x \\
& \leq C_{4}\left[\left(\int_{\Omega}\left|\xi_{n}\right|^{\delta} \varphi_{1}^{\delta} \frac{\left|w_{n}\right|^{\frac{1}{1-\mu}}}{\varphi_{1}^{\frac{\mu}{1-\mu}}} d x\right)^{1-\mu}+\left(\int_{\Omega} \frac{\left|w_{n}\right|^{\delta+\frac{1}{1-\mu}}}{\varphi_{1}^{\frac{\mu}{1-\mu}}} d x\right)^{1-\mu}\right] \\
& +\int_{\Omega} \gamma_{1} w_{n} d x \\
& \leq C_{4}\left|\xi_{n}\right|^{\delta(1-\mu)}\left(\int_{\Omega} \frac{\left|w_{n}\right|^{\frac{1}{1-\mu}}}{\varphi_{1}^{\frac{\mu}{1-\mu}-\delta}} d x\right)^{1-\mu}+C_{4}\left(\int_{\Omega} \frac{\left|w_{n}\right|^{\delta+\frac{1}{1-\mu}}}{\varphi_{1}^{\frac{\mu}{1-\mu}}} d x\right)^{1-\mu} \\
& +C_{5}\left\|w_{n}\right\|_{H_{0}^{1}} \text {. }
\end{aligned}
$$


By Hardy-Sobolev inequality [3, Lemma 2.2], we deduce

$$
\left\|w_{n}\right\|_{H_{0}^{1}} \leq C_{6}\left[\left|\xi_{n}\right|^{\delta(1-\mu)}+\left\|w_{n}\right\|_{H_{0}^{1}}^{\delta(1-\mu)}+1\right],
$$

and hence, as $\delta(1-\mu)<1$, we obtain

$$
\left\|w_{n}\right\|_{H_{0}^{1}} \leq C_{7}\left(\left|\xi_{n}\right|^{\delta(1-\mu)}+1\right)
$$

which proves Step 1.

Step 2. We have $\xi_{n} \rightarrow-\infty$. By (4.15) and a bootstrap argument, we have $\left|\xi_{n}\right| \rightarrow$ $\infty$ and as $u_{n} \leq \beta_{1}$ we obtain moreover

$$
\xi_{n} \int_{\Omega} \varphi_{1}^{2} d x=\int_{\Omega} u_{n} \varphi_{1} d x \leq \int_{\Omega} \beta_{1} \varphi_{1} d x,
$$

and hence $\xi_{n} \rightarrow-\infty$.

Step 3. For $n$ large enough, $u_{n} \leq \beta_{2}$. Observe that this claim contradicts the localization $u_{n} \notin C^{\beta_{2}} \cup C_{\alpha_{1}}$ and hence proves the claim.

Recall that $\beta_{2}=-\epsilon \varphi_{1}$ is such that, for all $r>\delta$,

$$
\begin{aligned}
& -\Delta \beta_{2}=f_{r}\left(x, \beta_{2}\right)+q(x), \text { in } \Omega, \\
& \beta_{2}=0, \quad \text { on } \partial \Omega \text {, }
\end{aligned}
$$

where $q(x) \geq\left(\lambda-\lambda_{1}\right) \epsilon \varphi_{1}(x)$.

Multiplying (4.13) by $\left(u_{n}-\beta_{2}\right)^{+}$and integrating we obtain

$$
\begin{aligned}
\int_{\Omega}\left|\nabla\left(u_{n}-\beta_{2}\right)^{+}\right|^{2} & =\int_{\Omega}\left[f_{r_{n}}\left(x, u_{n}\right)-f_{r_{n}}\left(x, \beta_{2}\right)-q(x)\right]\left(u_{n}-\beta_{2}\right)^{+} d x \\
& \leq \int_{\Omega}\left[f_{r_{n}}\left(x, u_{n}\right)-f_{r_{n}}\left(x, \beta_{2}\right)-q(x)\right]^{+}\left(u_{n}-\beta_{2}\right)^{+} d x .
\end{aligned}
$$

Hence, denoting by $\chi_{A}$ the characteristic function of the set $A$ and using the Sobolev injections, we have that, for every $q^{\prime}>\frac{2 N}{N+2}$,

$$
\left\|\left(u_{n}-\beta_{2}\right)^{+}\right\|_{H_{0}^{1}} \leq C_{8}\left\|\left[f_{r_{n}}\left(x, u_{n}\right)-f_{r_{n}}\left(x, \beta_{2}\right)-q(x)\right]^{+} \chi_{\left\{u_{n} \geq \beta_{2}\right\}}\right\|_{L^{q^{\prime}}} .
$$

As $u_{n} \leq \beta_{1}$ we obtain

$$
\begin{aligned}
{\left[f_{r_{n}}\left(x, u_{n}\right)-f_{r_{n}}\left(x, \beta_{2}\right)-q(x)\right]^{+} \chi_{\left\{u_{n} \geq \beta_{2}\right\}} } & \leq\left[f_{r_{n}}\left(x, \beta_{2}+\left(u_{n}-\beta_{2}\right)^{+}\right)\right. \\
& \left.-f_{r_{n}}\left(x, \beta_{2}\right)-q(x)\right]^{+} \\
& \leq\left[\lambda_{1}\left(\beta_{1}-\beta_{2}\right)-\left|\beta_{2}\right|^{\delta}+2 \gamma_{1}-q\right]^{+}
\end{aligned}
$$

Combining (4.16) with (4.17), we deduce that

$$
\left\|\left(u_{n}-\beta_{2}\right)^{+}\right\|_{H_{0}^{1}} \leq C_{9} .
$$

Moreover we have, for $n$ large enough,

$$
\frac{u_{n}-\beta_{2}}{\left|\xi_{n}\right|}=-\varphi_{1}+\frac{w_{n}-\beta_{2}}{\left|\xi_{n}\right|} \text {. }
$$


As $w_{n} /\left|\xi_{n}\right| \rightarrow 0$ in $H_{0}^{1}(\Omega)$ we deduce that

$$
\frac{u_{n}-\beta_{2}}{\left|\xi_{n}\right|} \rightarrow-\varphi_{1} \quad \text { a.e. in } \Omega,
$$

which means that, for a.e. $x \in \Omega$, there exists $N_{x}>0$ such that, for all $n \geq N_{x}$, $\left(u_{n}-\beta_{2}\right)(x) \leq 0$ and hence $\left(u_{n}-\beta_{2}\right)^{+}(x)=0$. This implies that

$$
\left(u_{n}-\beta_{2}\right)^{+}(x) \rightarrow 0 \quad \text { a.e. in } \Omega \text {. }
$$

By the first inequality of (4.17) we obtain

$$
\left[f_{r_{n}}\left(x, u_{n}\right)-f_{r_{n}}\left(x, \beta_{2}\right)-q(x)\right]^{+} \chi_{\left\{u_{n} \geq \beta_{2}\right\}} \rightarrow 0, \quad \text { a.e. in } \Omega,
$$

and by the second inequality of (4.17) we prove that

$$
\left[f_{r_{n}}\left(x, u_{n}\right)-f_{r_{n}}\left(x, \beta_{2}\right)-q(x)\right]^{+} \chi_{\left\{u_{n} \geq \beta_{2}\right\}} \rightarrow 0, \quad \text { in } L^{q^{\prime}}(\Omega),
$$

for $q^{\prime}<p$. Using (4.16), we conclude that

$$
\left\|\left(u_{n}-\beta_{2}\right)^{+}\right\|_{H_{0}^{1}} \rightarrow 0 \quad \text { as } n \rightarrow \infty .
$$

Let us prove now that

$$
\lim _{n \rightarrow \infty} \frac{\left\|\left[f_{r_{n}}\left(x, u_{n}\right)-f_{r_{n}}\left(x, \beta_{2}\right)-q(x)\right]^{+} \chi_{\left\{u_{n} \geq \beta_{2}\right\}}\right\|_{L^{q^{\prime}}}}{\left\|\left(u_{n}-\beta_{2}\right)^{+}\right\|_{H_{0}^{1}}}=0 .
$$

Recall that, for a.e. $x \in \Omega$, there exists $N_{x}>0$ such that, for all $n \geq N_{x}$, $\left[f_{r_{n}}\left(x, u_{n}\right)-f_{r_{n}}\left(x, \beta_{2}\right)-q(x)\right]^{+} \chi_{\left\{u_{n} \geq \beta_{2}\right\}}=0$ and hence

$$
\lim _{n \rightarrow \infty} \frac{\left[f_{r_{n}}\left(x, u_{n}(x)\right)-f_{r_{n}}\left(x, \beta_{2}(x)\right)-q(x)\right]^{+} \chi_{\left\{u_{n} \geq \beta_{2}\right\}}(x)}{\left\|\left(u_{n}-\beta_{2}\right)^{+}\right\|_{H_{0}^{1}}}=0, \quad \text { a.e. in } \Omega \text {. }
$$

Moreover, using the definition of $f_{r}$, we have that a.e. in $\Omega$,

$$
\frac{\left[f_{r_{n}}\left(x, u_{n}\right)-f_{r_{n}}\left(x, \beta_{2}\right)-q(x)\right]^{+} \chi_{\left\{u_{n} \geq \beta_{2}\right\}}}{\left\|\left(u_{n}-\beta_{2}\right)^{+}\right\|_{H_{0}^{1}}} \leq \frac{\left[f\left(x, \beta_{2}+\left(u_{n}-\beta_{2}\right)^{+}\right)-f\left(x, \beta_{2}\right)-q(x)\right]^{+}}{\left\|\left(u_{n}-\beta_{2}\right)^{+}\right\|_{H_{0}^{1}}} .
$$

The function $f$ being locally $L^{\infty}$-Lipschitz in $u$ uniformly in $x$ and using the inequality $u_{n} \leq \beta_{1}$, we obtain

$$
\left|f\left(x, \beta_{2}+\left(u_{n}-\beta_{2}\right)^{+}\right)-f\left(x, \beta_{2}\right)\right| \leq C_{10}\left|\left(u_{n}-\beta_{2}\right)^{+}\right| .
$$

As $\frac{\left(u_{n}-\beta_{2}\right)^{+}}{\left\|\left(u_{n}-\beta_{2}\right)^{+}\right\|_{H_{0}^{1}}}$ is bounded in $H_{0}^{1}(\Omega)$, up to a subsequence, for all $q<\frac{2 N}{N-2}$, there exists $\gamma_{2} \in L^{q}(\Omega)$ such that, a.e. in $\Omega$,

$$
\frac{\left[f\left(x, \beta_{2}+\left(u_{n}-\beta_{2}\right)^{+}\right)-f\left(x, \beta_{2}\right)-q(x)\right]^{+}}{\left\|\left(u_{n}-\beta_{2}\right)^{+}\right\|_{H_{0}^{1}}} \leq C_{10} \frac{\left|\left(u_{n}-\beta_{2}\right)^{+}\right|}{\left\|\left(u_{n}-\beta_{2}\right)^{+}\right\|_{H_{0}^{1}}} \leq C_{10} \gamma_{2} .
$$

Hence as $q>2>q^{\prime}$ we have, by the Lebesgue convergence theorem,

$$
\lim _{n \rightarrow \infty} \frac{\left\|\left[f_{r_{n}}\left(x, u_{n}\right)-f_{r_{n}}\left(x, \beta_{2}\right)-q(x)\right]^{+} \chi_{\left\{u_{n} \geq \beta_{2}\right\}}\right\|_{L^{q^{\prime}}}}{\left\|\left(u_{n}-\beta_{2}\right)^{+}\right\|_{H_{0}^{1}}}=0,
$$

which implies by (4.16) that, for $n$ large enough, $\left\|\left(u_{n}-\beta_{2}\right)^{+}\right\|_{H_{0}^{1}}=0$ and proves Step 3. As this contradicts the localization, Claim 1 is proved. 
Let $R>K_{1}$ be fixed and consider the modified corresponding problem

$$
\begin{array}{cc}
-\Delta u=f_{R}(x, u), & \text { in } \Omega, \\
u=0, & \text { on } \partial \Omega .
\end{array}
$$

Observe that, there exists $\gamma_{3} \in L^{p}(\Omega)$ such that, for a.e. $x \in \Omega$ and all $u \leq \beta_{1}(x)$, we have

$$
\left|f_{R}(x, u)-\lambda_{1} u\right| \leq \gamma_{3}(x) .
$$

Part 2-Second modified problem. For every $s>R$, define the function $\tilde{f}_{s}: \Omega \times$ $\mathbb{R} \rightarrow \mathbb{R}$ by

$$
\begin{aligned}
\tilde{f}_{s}(x, u) & =\left(\lambda_{1}-\frac{1}{s}\right) u, & & \text { if } u<-s-1, \\
& =-(u+s)\left(\lambda_{1}-\frac{1}{s}\right) u+(s+1+u) f_{R}(x, u) & & \text { if }-s-1 \leq u \leq-s, \\
& =f_{R}(x, u), & & \text { if } u>-s .
\end{aligned}
$$

For every $s>R$, consider the modified problem

$$
\begin{array}{cc}
-\Delta u=\tilde{f}_{s}(x, u), & \text { in } \Omega, \\
u=0, & \text { on } \partial \Omega .
\end{array}
$$

Observe that we can decompose $\tilde{f}_{s}(x, u)=p_{s}(x, u) u+q_{s}(x, u)$ such that, for a.e. $x \in \Omega$ and all $u \leq \beta_{1}(x)$,

$$
\begin{gathered}
\lambda_{1}-\frac{1}{s} \leq p_{s}(x, u) \leq \lambda_{1} \\
\left|q_{s}(x, u)\right| \leq \gamma_{3}(x)
\end{gathered}
$$

Moreover, as previously, for all $s>R$, every solution $u$ of (4.19) satisfies $u \leq \beta_{1}$.

Claim 2: There exists $K_{2}>R$ such that, for all $s>K_{2}$ and for all $u \notin C^{\beta_{2}} \cup C_{\alpha_{1}}$ solution of (4.19), we have $\|u\|_{\mathcal{C}^{1}}<K_{2}$. Otherwise, for all $n \geq 1$, there exist $s_{n}>n$ and $u_{n} \notin C^{\beta_{2}} \cup C_{\alpha_{1}}$ solution of (4.19) for $s=s_{n}$ with $\left\|\bar{u}_{n}\right\|_{\mathcal{C}^{1}} \geq n$. Then $v_{n}=u_{n} /\left\|u_{n}\right\|_{\mathcal{C}^{1}}$ satisfies

$$
\begin{array}{cc}
-\Delta v_{n}=p_{s_{n}}\left(x, u_{n}\right) v_{n}+\frac{q_{s_{n}}\left(x, u_{n}\right)}{\left\|u_{n}\right\|_{\mathcal{C}^{1}}}, & \text { in } \Omega, \\
v_{n}=0, & \text { on } \partial \Omega .
\end{array}
$$

As $\left\{p_{s_{n}}\left(x, u_{n}\right) v_{n}+\frac{q_{s_{n}}\left(x, u_{n}\right)}{\left\|u_{n}\right\|_{\mathcal{C}^{1}}} \mid n \in \mathbb{N}\right\}$ is bounded in $L^{p}(\Omega)$, we deduce that, up to a subsequence, $v_{n_{k}} \rightarrow v$ in $\mathcal{C}^{1}(\bar{\Omega})$. It is then easy to see that $p_{s_{n_{k}}}\left(x, u_{n_{k}}\right) v_{n_{k}}+$ $\frac{q_{s_{n_{k}}}\left(x, u_{n_{k}}\right)}{\left\|u_{n_{k}}\right\|_{\mathcal{C}^{1}}} \rightarrow \lambda_{1} v$ in $L^{p}(\Omega)$. Passing to the limit, $v$ is a solution of

$$
\begin{array}{cc}
-\Delta v=\lambda_{1} v, & \text { in } \Omega, \\
v=0, & \text { on } \partial \Omega, \\
v \leq 0, & \text { in } \Omega .
\end{array}
$$

We deduce that $v=-\varphi_{1}$. Hence for $k$ large enough, using [16, Lemma 3.1],

$$
u_{n_{k}} \leq-\frac{1}{2}\left\|u_{n_{k}}\right\|_{\mathcal{C}^{1} \varphi_{1}} \ll \beta
$$

which contradicts the localization of $u_{n_{k}}$ and proves the claim. 
Conclusions - Let $s>K_{2}$ be fixed and consider the modified functional

$$
\phi_{s}(u)=\int_{\Omega}\left[\frac{|\nabla u(x)|^{2}}{2}-\tilde{F}_{s}(x, u(x))\right] d x,
$$

where $\tilde{F}_{s}(x, u)=\int_{0}^{u} \tilde{f}_{s}(x, \xi) d \xi$.

Observe that every critical point $u$ of $\phi_{s}$ is a solution of (4.19). Moreover, if $u \notin C^{\beta_{2}} \cup C_{\alpha_{1}}$, by Claim 2, $u$ is a solution of (4.18) and by Claim 1, it is a solution of (1.1). We also see that the problem (4.19) has a lower solution $\alpha_{2} \ll \beta_{2}$. In fact, let $w \in W^{2, p}(\Omega)$ be the solution of

$$
\begin{array}{cc}
-\Delta w=\left(\lambda_{1}-\frac{1}{s}\right) w-\left(\lambda_{1}-\frac{1}{s}\right)(s+2), & \text { in } \Omega, \\
w=0, & \text { on } \partial \Omega .
\end{array}
$$

Choose $k>0$ large enough such that $\alpha_{2}:=w-k \varphi_{1}-s-2 \leq-s-2$. Hence it is easy to see that $\alpha_{2}$ is a lower solution of (4.19) with $\alpha_{2} \ll \beta_{2}$.

The rest of the proof is similar to the one of Theorems 4.1 and 4.2 respectively.

Remark 4.2. Observe that we can replace the minoration on $f$ in $(\mathrm{v})$ by

$$
\liminf _{u \rightarrow-\infty} \frac{f(x, u)}{|u|^{\frac{N+1}{N-1}}}=0, \quad \text { uniformly in } x .
$$

In the ordinary differential case

$$
\begin{gathered}
-u^{\prime \prime}=f(t, u), \\
u(0)=0, u(T)=0,
\end{gathered}
$$

we can improve the above results by requiring just a lower bound on $f(t, u) / u$ for $u \rightarrow-\infty$. We obtain the following theorem.

Theorem 4.5. Let $f:[0, T] \times \mathbb{R} \rightarrow \mathbb{R}:(t, u) \mapsto f(t, u)$ be an $L^{1}$-Carathéodory function, locally $L^{\infty}$-Lipschitz in $u$ uniformly in $t$. Assume

(i) there exist $\lambda>(2 \pi / T)^{2}$ and $\delta>0$ such that for a.e. $t \in[0, T]$ and all $u \in[-\delta, \delta] \backslash\{0\}$,

$$
\frac{f(t, u)}{u} \geq \lambda
$$

(ii) there exist $\mu<(\pi / T)^{2}$ and $R>0$ such that for a.e. $t \in[0, T]$ and all $u \geq R$,

$$
\frac{f(t, u)}{u} \leq \mu
$$

(vi) there exist a function $a \in L^{1}(0, T)$ and $R^{\prime}>0$ such that for a.e. $t \in[0, T]$ and all $u \leq-R^{\prime}$,

$$
\frac{f(t, u)}{u} \geq a(t) .
$$


Then the problem (4.20) has at least two nontrivial solutions: one positive and the other one sign-changing.

If moreover $f \in \mathcal{C}^{1}([0, T] \times \mathbb{R})$ and there exist $p, q, k \geq 2(k \in \mathbb{N})$ and $\delta>0$ such that for a.e. $t \in[0, T]$ and all $u \in[-\delta, \delta] \backslash\{0\}$,

$$
\frac{k^{2} \pi^{2}}{T^{2}}<p \leq \frac{f(t, u)}{u} \leq q<\frac{(k+1)^{2} \pi^{2}}{T^{2}},
$$

then the problem (4.20) has a second sign-changing solution.

Proof. The only modification concerns the proof of the Step 6 in the proof of Theorem 4.1. Consider the modified problem

$$
\begin{gathered}
-u^{\prime \prime}=f_{r}(t, u), \\
u(0)=0, u(T)=0,
\end{gathered}
$$

where

$$
\begin{aligned}
f_{r}(t, u) & =0, & & \text { if } u<-r-1, \\
& =(1+r+u) f(t, u), & & \text { if }-r-1 \leq u<-r, \\
& =f(t, u), & & \text { if }-r \leq u \leq \beta_{1}(t), \\
& =f\left(t, \beta_{1}(t)\right), & & \text { if } u>\beta_{1}(t) .
\end{aligned}
$$

By the maximum principle as in Theorem 3.2 we have that every solution $u$ of (4.21) satisfies $u \leq \beta_{1}$.

Claim: There exists $K>0$ such that for all $r>K$ and all solutions $u$ of (4.21) with $u \notin C^{\beta_{2}} \cup C_{\alpha_{1}}$ we have $\|u\|_{\mathcal{C}^{1}}<K$. As $u \notin C^{\beta_{2}} \cup C_{\alpha_{1}}$, there exists $t_{1} \in[0, T]$ such that $u\left(t_{1}\right) \geq \beta_{2}\left(t_{1}\right)$ and $u^{\prime}\left(t_{1}\right)=\beta_{2}^{\prime}\left(t_{1}\right)$.

Let $\hat{a} \in L^{1}\left(0, T ; \mathbb{R}^{+}\right)$and $h \in L^{1}(0, T)$ such that, for a.e. $t \in[0, T]$, all $u \leq \beta_{1}(t)$ and all $r>\left\|\beta_{2}\right\|_{\infty}$,

$$
f_{r}(t, u) \leq \hat{a}(t)|u|+h(t)
$$

Hence we have

$$
\begin{aligned}
u(t) & =u\left(t_{1}\right)+u^{\prime}\left(t_{1}\right)\left(t-t_{1}\right)-\int_{t_{1}}^{t}(t-s) f_{r}(s, u(s)) d s \\
& \geq \beta_{2}\left(t_{1}\right)+\beta_{2}^{\prime}\left(t_{1}\right)\left(t-t_{1}\right)-\int_{t_{1}}^{t}(t-s)[\hat{a}(s)|u(s)|+h(s)] d s \\
& \geq-\epsilon-\epsilon \frac{\pi}{T}-T\|h\|_{L^{1}}-\int_{t_{1}}^{t}(t-s) \hat{a}(s)|u(s)| d s
\end{aligned}
$$

i.e. we have $C>0$ such that, for all $t \in[0, T]$,

$$
|u(t)| \leq C+\int_{t_{1}}^{t} T \hat{a}(s)|u(s)| d s .
$$

The Claim follows from Gronwall's Lemma.

The rest of the proof follows as in Theorems 4.1 and 4.2 respectively.

As in Theorem 4.3, in case $f$ is independent of $t$, the condition (vi) is not needed. 
Theorem 4.6. Let $f: \mathbb{R} \rightarrow \mathbb{R}: u \mapsto f(u)$ be a locally Lipschitz continuous function. Assume

(i) there exist $\lambda>(2 \pi / T)^{2}$ and $\delta>0$ such that for all $u \in[-\delta, \delta] \backslash\{0\}$,

$$
\frac{f(u)}{u} \geq \lambda
$$

(ii) there exist $\mu<(\pi / T)^{2}$ and $R>0$ such that for all $u \geq R$,

$$
\frac{f(u)}{u} \leq \mu \text {. }
$$

Then the problem

$$
\begin{gathered}
-u^{\prime \prime}=f(u), \\
u(0)=0, u(T)=0,
\end{gathered}
$$

has at least two nontrivial solutions: one positive and the other one sign-changing. If moreover $f \in \mathcal{C}^{1}(\mathbb{R})$ and there exist $p, q, k \geq 2(k \in \mathbb{N})$ and $\delta>0$ such that for all $u \in[-\delta, \delta] \backslash\{0\}$,

$$
\left(\frac{k \pi}{T}\right)^{2}<p \leq \frac{f(u)}{u} \leq q<\left(\frac{(k+1) \pi}{T}\right)^{2},
$$

then the problem (4.22) has a second sign-changing solution.

Remark 4.3. Remark that in all the results of this section, the condition (ii) was just used to construct the upper solution $\beta_{1}$. Hence we can replace (ii) by requiring the existence of such an upper solution $\beta_{1} \gg 0$ or by any condition ensuring this existence.

\section{Acknowledgment}

The author wishes to thank Patrick Habets for fruitful discussions.

\section{References}

[1] Amann, H.: A note on degree theory for gradient mappings. Proc. A.M.S. 85, 591-595 (1982)

[2] Bartsch, T., Wang, Z.Q.: On the existence of sign-changing solutions for semilinear Dirichlet problems. Top. Meth. Nonlinear Anal. 7, 115-131 (1996)

[3] Brezis, H., Turner, R.E.L.: On a class of superlinear elliptic problems. Comm. Partial Differ. Equ. 2, 601-614 (1977)

[4] Chang, K.C.: A variant mountain pass lemma. Sci. Sin. A 26, 1241-1255 (1983)

[5] Chang, K.C.: Variational methods and sub- and super-solutions. Sci. Sin. A 26, 1256-1265 (1983) 
[6] Chang, K.C.: Infinite Dimensional Morse Theory and Multiple Solution Problems. Birkhäuser, Boston (1993)

[7] Conti, M., Merizzi, L., Terracini, S.: Remarks on variational methods and lowerupper solutions. Nonlinear Differ. Equ. Appl. 6, 371-393 (1999)

[8] Dancer, E.N., Du, Y.: Multiple solutions of some semilinear elliptic equations via the generalized Conley index. J. Math. Anal. Appl. 189, 848-871 (1995)

[9] Dancer, E.N., Du, Y.: On sign-changing solutions of certain semilinear elliptic problems. Appl. Anal. 56, 195-206 (1995)

[10] Dancer, E.N., Du, Y.: A note on multiple solutions of some semilinear elliptic problems. J. Math. Anal. Appl. 211, 626-640 (1997)

[11] De Coster, C., Habets, P.: Two-point boundary value problems: lower and upper solutions. Mathematics in Science and Engineering 205. Elsevier, Amsterdam (2006)

[12] De Coster, C., Henrard, M.: Existence and localization of solution for elliptic problem in presence of lower and upper solutions without any order. J. Differ. Equ. 145, 420-452 (1998)

[13] de Figueiredo, D.G.: Lectures on the Ekeland variational principle with applications and detours. Tata Institute of Fundamental Research, Lectures on Math. and Phys. 81, Springer Verlag, Berlin-New York (1989)

[14] de Figueiredo, D.G., Lions, P.L., Nussbaum, R.D.: A priori estimates and existence of positive solutions of semilinear elliptic equations. J. Math. Pures Appl. 61, 41-63 (1982)

[15] Gidas, B., Spruck, J.: A priori bounds for positive solutions in nonlinear elliptic equations. Comm. Partial Differ. Equ. 6, 883-901 (1981)

[16] Habets, P., Omari, P.: Existence and localization of solutions of second order elliptic problems using lower and upper solutions in the reversed order. Topol. Methods Nonlinear Anal. 8, 25-56 (1996)

[17] Höfer, H.: Variational and topological methods in partially ordered Hilbert spaces. Math. Ann. 261, 493-514 (1982)

[18] Höfer, H.: A note on the topological degree at a critical point of the Mountain-Pass type. Proc. A.M.S. 90, 309-315 (1984)

[19] Li, S.J., Wang, T.: Mountain pass theorem in order intervals and multiple solutions for semilinear elliptic Dirichlet problems. J. Anal. Math. 81, 373-396 (2000)

[20] Li, S., Zhang, Z.: Sign-changing and multiple solutions theorems for semilinear elliptic boundary value problems with jumping nonlinearities. Acta Math. Sin. (English Series) 16, 113-122 (2000)

[21] Liu, Z.: Positive solutions of superlinear elliptic equations. J. Funct. Anal. 167, 370-398 (1999) 
[22] Liu, Z.: Multiplicity of solutions of semilinear elliptic boundary value problems with jumping nonlinearities at zero. Nonlinear Anal. A: Theory Methods 48, 1051-1063 (2002)

[23] Liu, Z., Li, S.: Contractibility of level sets of functional associated with some elliptic boundary value problems and applications. Nonlinear Differ. Equ. Appl. 10, 133-170 (2003)

[24] Liu, Z., Sun, J.: Calculus of variations and super and sub-solutions in reversed order. Acta Math. Sin. (in Chinese) 37, 512-514 (1994)

[25] Liu, Z., Sun, J.: Invariant sets of descending flow in critical point theory with applications to nonlinear differential equations. J. Differ. Equ. 172, 257-299 (2001)

[26] Liu, Z., Sun, J.: An elliptic problem with jumping nonlinearities. Nonlinear Anal. 63, 1070-1082 (2005)

[27] Lloyd, N.G.: Degree Theory. Cambridge University Press, Cambridge (1978)

[28] Mawhin, J., Willem, M.: Critical Point Theory and Hamiltonian Systems. SpringerVerlag, Berlin (1989)

[29] Rouche, N., Mawhin, J.: Equations différentielles ordinaires. Masson, Paris (1973)

[30] Troianiello, G.M.: Elliptic Differential Equations and Obstacle Problems. Plenum Press, New York (1987)

C. De Coster $(\bowtie)$

Univ Lille Nord de France,

59000 Lille, France

e-mail: colette.de.coster@lmpa.univ-littoral.fr

C. De Coster

ULCO, LMPA J. Liouville, B.P. 699, 62228 Calais, France

C. De Coster

CNRS, FR 2956, Paris, France

Received: 23 September 2008.

Accepted: 24 May 2009. 